\title{
ERRATUM
}

\section{Myxoma Virus Induces Type I Interferon Production in Murine Plasmacytoid Dendritic Cells via a TLR9/MyD88-, IRF5/IRF7-, and IFNAR-Dependent Pathway}

Peihong Dai, Hua Cao, Taha Merghoub, Francesca Avogadri, Weiyi Wang, Tanvi Parikh, Chee-Mun Fang, Paula M. Pitha, Katherine A. Fitzgerald, Masmudur M. Rahman, Grant McFadden, Xiaoyu Hu, Alan N. Houghton, Stewart Shuman, and Liang Deng

Dermatology Service, Department of Medicine, Molecular Biology Program, and Immunology Program, Memorial Sloan-Kettering Cancer Center, New York, New York 10065; Weill Medical College of Cornell University, New York, New York 10021; Department of Biology, The Johns Hopkins University, Baltimore, Maryland 21218; Division of Infectious Diseases and Immunology, Department of Medicine, University of Massachusetts Medical School, Worcester, Massachusetts 01605;

Department of Molecular Genetics and Microbiology, College of Medicine, University of Florida, Gainesville, Florida 32610; and Laboratory of Cellular Signaling and Immune Regulation, Hospital for Special Surgery, New York, New York 10021

Volume 85 , no. 20, p. 10814-10825, 2011. Page 10818, Fig. 3: Delete "(\% of control)" from the $y$ axis. The $y$ axis represents relative mRNA expression compared with no virus control. 